\title{
Monte Carlo study of the two-dimensional kinetic Blume-Capel model in a quenched random crystal field
}

\author{
Alexandros Vasilopoulos ${ }^{1}$, Zeynep Demir Vatansever ${ }^{2}$, \\ Erol Vatansever ${ }^{1,2}$, and Nikolaos G. Fytas ${ }^{1 *}$ \\ ${ }^{1}$ Centre for Fluid and Complex Systems, Coventry University, \\ Coventry, CV1 5FB, United Kingdom and \\ ${ }^{2}$ Department of Physics, Dokuz Eylül University, TR-35160, Izmir, Turkey
}

(Dated: July 20, 2021)

\begin{abstract}
We investigate by means of Monte Carlo simulations the dynamic phase transition of the twodimensional kinetic Blume-Capel model under a periodically oscillating magnetic field in the presence of a quenched random crystal-field coupling. We analyze the universality principles of this dynamic transition for various values of the crystal-field coupling at the originally second-order regime of the corresponding equilibrium phase diagram of the model. A detailed finite-size scaling analysis indicates that the observed nonequilibrium phase transition belongs to the universality class of the equilibrium Ising ferromagnet with additional logarithmic corrections in the scaling behavior of the heat capacity. Our results are in agreement with earlier works on kinetic Ising models.
\end{abstract}

\footnotetext{
* Corresponding author: nikolaos.fytas@coventry.ac.uk
} 


\section{INTRODUCTION}

In the last decades our understanding of equilibrium critical phenomena has developed to a point where well-established results are available for a wide variety of systems. In particular, the origin and/or the difference between equilibrium universality classes is by now well understood. This observation also partially holds for systems under the presence of quenched disorder. However, far less is known for the physical mechanisms underlying the nonequilibrium phase transitions of many-body interacting systems that are far from equilibrium and clearly a solid classification of nonequilibrium phase transitions into universality classes is missing.

We know today that when a ferromagnetic system, below its Curie temperature, is exposed to a time-dependent oscillating magnetic field, it may exhibit a fascinating dynamical behavior [1]. In a typical ferromagnetic system being subjected to an oscillating magnetic field, there occurs a competition between the time scales of the half period of the applied field $t_{1 / 2}$ and the metastable lifetime, $\tau$, which is defined as the average time it takes the system to leave one of its two degenerate zero-field equilibrium states when a field of magnitude $h_{0}$ opposite to the initial magnetization is applied. In practice, $\tau$ is measured as the first-passage time to zero magnetization. When $t_{1 / 2}<\tau$, the time-dependent magnetization tends to oscillate around a nonzero value which corresponds to the dynamically ordered phase. In this region, the time-dependent magnetization is not capable of following the external field. However, for larger values of the half period, the system is given enough time to follow the external field and in this case the time-dependent magnetization oscillates around its zero value, indicating a dynamically disordered phase. When $t_{1 / 2} \approx \tau$, a dynamic phase transition takes place between the dynamically ordered and disordered phases.

Throughout the years, there have been several theoretical [2 22] and experimental studies [23 27] dealing with dynamic phase transitions and hysteresis phenomena. The main conclusion is that both the amplitude and period of the time-dependent magnetic field play a key role in dynamic critical phenomena. Furthermore, the characterization of universality classes in spin models driven by a time-dependent oscillating magnetic field has also attracted a lot of interest lately [28 37]. A short listing of the main results is given below:

(1) The critical exponents of the kinetic Ising model were found to be compatible to those of the equilibrium Ising model at both two (2D) and three dimensions (3D) [28, 30, 32. 
(2) Buendía and Rikvold [31] estimated the critical exponents of the 2D Ising model and provided strong evidence that the characteristics of dynamic phase transition are universal with respect to the choice of stochastic dynamics. These authors used the so-called soft Glauber dynamics [38], for which both nucleation and interface propagation are slower and the interfaces smoother than for the standard hard Glauber and Metropolis dynamics.

(3) The role of surfaces at nonequilibrium phase transitions in Ising models has been elucidated by Park and Pleimling [33]: The nonequilibrium surface exponents were found to be different than their equilibrium counterparts.

(4) Experimental evidence by Riego et al. [27] and numerical results by Buendía and Rikvold [35] verified that the equivalence of the dynamic phase transition to an equilibrium phase transition is limited to the area near the critical period and for zero bias.

(5) Numerical simulations by Vatansever and Fytas showed that the nonequilibrium phase transitions of the pure and random-bond spin-1 Blume-Capel model belong to the universality class of the equilibrium pure Ising model with logarithmic corrections in the disordered case [36, 37]. Some general and very useful features of the dynamic phase transition of the pure Blume-Capel model can also be found in Refs. [8, 16, 17, 19, 39, 40].

The above results in 2D and 3D kinetic Ising and Blume-Capel models establish a mapping between the universality principles of the equilibrium and dynamic phase transitions of spin-1/2 and spin-1 models. They also provide additional support in favor of an earlier investigation of a Ginzburg-Landau model with a periodically changing field [10], as well with the symmetry-based arguments of Grinstein et al. in nonequilibrium critical phenomena [41].

As the vast majority of works in the field deal with pure systems, in the present paper we attempt to shed some additional light on the effect of quenched disorder on dynamic phase transitions [37]. We should note here some earlier mean-field and effective-field theory treatments of the problem where it was shown that the dynamic character of a typical system driven by a time-dependent magnetic field sensitively depends on the amount of disorder, accounting for re-entrant phenomena and dynamic tricritical points [42 47].

In this paper we use as a test-case platform for our numerical experiment the squarelattice Blume-Capel model [48] under a time-dependent magnetic field, diffusing disorder in the crystal-field coupling [see below Eq. (2)]. This type of randomness has also been used in the past for the equilibrium version of the model by Branco and Boechat [49], Sumedha and Mukherjee [50], Vatansever et al. [51] and is much closer to the experimental reality 
as it mimics the physics of random porous media in ${ }^{3} \mathrm{He}-{ }^{4} \mathrm{He}$ mixtures [52]. In a nutshell, our extensive Monte-Carlo simulations for various values of the crystal-field coupling along the phase boundary indicate that the dynamic phase transition of the model belongs to the universality class of the corresponding equilibrium Ising model with logarithmic corrections in the heat-capacity scaling due to the presence of quenched disorder.

The rest of the paper is organized as follows: In Sec. II we introduce the model, provide details of our simulation protocol and define the relevant thermodynamic observables. The numerical results are presented in Sec. III and a summary of our conclusions is given in Sec. IV.

\section{MODEL AND METHODS}

\section{A. Model}

The Hamiltonian of the Blume-Capel model under a time-dependent oscillating magnetic field reads as

$$
\mathcal{H}=-J \sum_{\langle x y\rangle} \sigma_{x} \sigma_{y}+\sum_{x} \Delta_{x} \sigma_{x}^{2}-h(t) \sum_{x} \sigma_{x}
$$

where the spin variable $\sigma_{x}$ takes on the values $\{-1,0,+1\},\langle x y\rangle$ indicates summation over nearest neighbors on the square lattice and the coupling $J>0$ denotes the ferromagnetic exchange interactions. $\Delta_{x}$ represents the crystal-field strength and controls the density of vacancies $\left(\sigma_{x}=0\right)$. As mentioned above we choose a site-dependent bimodal crystal-field probability distribution of the form

$$
\mathcal{P}\left(\Delta_{x}\right)=p \delta\left(\Delta_{x}+\Delta\right)+(1-p) \delta\left(\Delta_{x}-\Delta\right)
$$

where $p \in(0,1)$ is the control parameter of the disorder distribution with $\mu=\Delta(1-2 p)$ and $s=2 \Delta \sqrt{p(1-p)}$ the mean value and standard deviation of the distribution (2), respectively. Finally, the term $h(t)$ corresponds to a spatially uniform periodically oscillating magnetic field, so that all lattice sites are exposed to a square-wave magnetic field with amplitude $h_{0}$ and half period $t_{1 / 2}[3032$.

Some useful explanatory comments for the equilibrium $(h(t)=0)$ version of the model are in order: 
(1) For $\Delta=\infty$ the model is equivalent to the random site spin- $1 / 2$ Ising model, where sites are present or absent with probability $p$ or $1-p$, respectively [49].

(2) For $p=0$ the pure Blume-Capel model is recovered [53 56]. The phase diagram of the pure $(p=0)$ and random $(p=1 / 2)$ model in the $\Delta-T$ plane is shown in Fig. 1 including a variety of critical and transition points from the current literature. For small $\Delta$ there is a line of continuous transitions (in the Ising universality class) between the ferromagnetic and paramagnetic phases that crosses the $\Delta=0$ axis at $T_{0} \approx 1.693$ [54]. For large $\Delta$ the transition becomes discontinuous and it meets the $T=0$ line at $\Delta_{0}=z J / 2$ [48], where $z=4$ is the coordination number (as usual we set $J=k_{\mathrm{B}}=1$ to fix the temperature scale). The two line segments meet at a tricritical point $\left(\Delta_{\mathrm{t}} \approx 1.966, T_{\mathrm{t}} \approx 0.608\right)$ [55].

(3) With the inclusion of disorder $(p>0)$ the critical temperature of the system rises - see the yellow filled squares in Fig. 1. For further explanations and simple arguments explaining this behavior we refer the reader to Ref. [51].

\section{B. Numerical approach}

We performed Monte Carlo simulations with periodic boundary conditions using the single-site update Metropolis algorithm [57 59]. This approach, together with the stochastic Glauber dynamics [60], consists the standard recipe in kinetic Monte Carlo simulations [31. Let us briefly outline below the steps of our algorithm:

(1) A lattice site is selected randomly among the $N=L \times L$ options.

(2) The spin variable located at the selected site is flipped, keeping the other spins in the system fixed.

(3) The energy change originating from this spin flip operation is calculated using the Hamiltonian of Eq. (1) via $\Delta \mathcal{H}=\mathcal{H}_{\mathrm{a}}-\mathcal{H}_{\mathrm{o}}$, where $\mathcal{H}_{\mathrm{a}}$ denotes the system's energy after the trial switch of the selected spin and $\mathcal{H}_{\mathrm{o}}$ corresponds to the total energy of the old spin configuration. The probability to accept the proposed spin update is given by

$$
\Pi\left(\sigma_{x} \rightarrow \sigma_{x}^{\prime}\right)= \begin{cases}\exp \left(-\Delta \mathcal{H} / k_{\mathrm{B}} T\right), & \text { if } \mathcal{H}_{a} \geq \mathcal{H}_{o} \\ 1, & \text { if } \mathcal{H}_{\mathrm{a}}<\mathcal{H}_{\mathrm{o}}\end{cases}
$$

(4) If the energy is lowered, the spin flip is always accepted.

(5) If the energy is increased, a random number $R$ is generated, such that $0 \leq R<1$ : If 
$R$ is less than or equal to the calculated Metropolis transition probability the selected spin is flipped. Otherwise, the old spin configuration remains unchanged. Note that all transitions among the three spin states $\{-1,0,+1\}$ are allowed in our numerical protocol.

Using the above scheme we simulated the model of Eqs. (1) and (2) at $\Delta=0.5$, 1 , and $\Delta=2$, fixing the control parameter $p$ to the value $1 / 2$, guided by the analysis of Ref. [51]. System sizes varied within the range $L=32-512$ and for each linear size an average over 500 independent realizations of the disorder was performed. The first $10^{3}$ periods of the external field were discarded during the thermalization process and numerical data were collected and analyzed during the following $11 \times 10^{3}$ periods of the field. Note that the time unit in our simulations is one Monte Carlo step per site (MCSS) and that error bars were estimated using the jackknife method [59]. Appropriate choices of magnetic-field strength, $h_{0}=0.3$, and temperature, $T(\Delta)=0.8 \times T_{\mathrm{c}}(\Delta)$, ensured that the metastable decay of the system following field reversal occurs through nucleation and growth of many droplets of the stable phase, i.e., the multidroplet regime. This point was already emphasized by Sides et al. in 1998 [28] - see also Ref. [32]. Here, $T_{\mathrm{c}}(\Delta=0.5)=1.6854, T_{\mathrm{c}}(\Delta=1)=1.6473$, and $T_{\mathrm{c}}(\Delta=2)=1.4907$ are the equilibrium critical temperatures of the random $p=1 / 2$ Blume-Capel model defined in Eqs. (1) and (2) [51.

Finally a comment on the fitting process discussed below in Sec. III; We employed the standard $\chi^{2}$ goodness of fit test [61]. Specifically, the $Q$-value of our $\chi^{2}$-test is the probability

of finding a $\chi^{2}$ value which is even larger than the one actually found from our data. We consider a fit as being fair only if $10 \% \leq Q \leq 90 \%$.

\section{Observables}

In order to determine the universality aspects of the kinetic random Blume-Capel model, we consider the half-period dependencies of various thermodynamic observables. The main quantity of interest is the period-averaged magnetization

$$
Q_{L}=\frac{1}{2 t_{1 / 2}} \oint M(t) d t
$$

where the integration is performed over one cycle of the oscillating field. Given that for finite systems in the dynamically ordered phase the probability density of $Q_{L}$ becomes bimodal, one has to measure the average norm of $Q_{L}$ in order to capture symmetry breaking so 
that $\langle|Q|\rangle_{L}$ defines the dynamic order parameter of the system. In Eq. (4), $M(t)$ is the time-dependent magnetization per site

$$
M(t)=\frac{1}{N} \sum_{x=1}^{N} \sigma_{x}(t) .
$$

To characterize and quantify the transition using finite-size scaling arguments we must also define quantities analogous to the susceptibility in equilibrium systems. The scaled variance of the dynamic order parameter

$$
\chi_{L}^{Q}=N\left[\left\langle Q^{2}\right\rangle_{L}-\langle|Q|\rangle_{L}^{2}\right]
$$

has been suggested as a proxy for the nonequilibrium susceptibility, also theoretically justified via fluctuation-dissipation relations [18].

Similarly, one may also measure the scaled variance of the period-averaged energy

$$
\chi_{L}^{E}=N\left[\left\langle E^{2}\right\rangle_{L}-\langle E\rangle_{L}^{2}\right]
$$

so that $\chi_{L}^{E}$ can be considered as the respective heat capacity. Here $E$ denotes the cycleaveraged energy corresponding to the cooperative part of the Hamiltonian (1). With the help of $Q$ we may also define the fourth-order Binder cumulant [28, 29]

$$
U_{L}^{Q}=1-\frac{\left\langle|Q|^{4}\right\rangle_{L}}{3\left\langle|Q|^{2}\right\rangle_{L}^{2}},
$$

a very useful observable for the characterization of universality classes [62].

\section{RESULTS}

As a starting point let us describe shortly the mechanism underlying dynamic ordering in kinetic ferromagnets as depicted in Figs. 2-4 4 below. In all these plots results for a single realization of the disorder are shown of a system size $L=192$ and for $\Delta=1$. Similar results were obtained also for the other $\Delta$ values but are omitted for brevity.

Figure 2 presents the time evolution of the magnetization and Fig. 3 the period dependencies of the dynamic order parameter $Q$ of the kinetic random Blume-Capel model. For rapidly varying fields, Fig. 2(a), the magnetization does not have enough time to switch during a single half period and remains nearly constant for many successive field cycles, as also illustrated by the black line in Fig. 3. On the other hand, for slowly varying fields, 
Fig. 2(c), the magnetization follows the field, switching every half period, so that $Q \approx 0$, as also shown by the blue line in Fig. 3. Thus, whereas in the dynamically disordered phase the ferromagnet is able to reverse its magnetization before the field changes again, in the dynamically ordered phase this is not possible and therefore the time-dependent magnetization oscillates around a finite value. The competition between magnetic field and the metastable state is captured by the half period $t_{1 / 2}$ (or by the normalized parameter $\Theta=t_{1 / 2} / \tau[32]$ ). Obviously, $t_{1 / 2}$ plays the role of temperature in the equilibrium system. Now, the transition between the two regimes is characterized by strong fluctuations in $Q$, see Fig. 2(b) and the evolution of the red line in Fig. 3. This behavior is indicative of a dynamic phase transition and occurs for values of the half period close to the critical one $t_{1 / 2}^{\mathrm{c}}$ (otherwise when $\Theta \approx 1$ ). Of course, since the value $t_{1 / 2}=66$ MCSS used for this illustration is slightly above $t_{1 / 2}^{\mathrm{c}}=65.96(6)$, see also Fig. 11 below, the observed behavior includes as well some nonvanishing finite-size effects.

Some additional spatial aspects of the transition scenarios described above via the configurations of a local order parameter $\left\{Q_{x}\right\}$ are shown in Fig. 4 . Below $t_{1 / 2}^{\mathrm{c}}$, see panel (a), the majority of spins spend most of their time in the +1 state, i.e., in the metastable phase during the first half period, and in the stable equilibrium phase during the second half period, except for fluctuations. Thus, most of the $Q_{x} \approx+1$ and the system lies in the dynamically ordered phase. On the other hand, when the period of external field is selected to be bigger than the relaxation time of the system, above $t_{1 / 2}^{\mathrm{c}}$, see panel (c), the system follows the field in every half period with some phase lag, and $Q_{x} \approx 0$ at all sites $x$. The system in this case is in the dynamically disordered phase. Near $t_{1 / 2}^{\mathrm{c}}$ and the expected dynamic phase transition, there are large clusters of both $Q_{x} \approx+1$ and -1 values within a sea of $Q_{x} \approx 0$, as shown in panel (b).

At this point we would like to scrutinize the effects of the zero spin state $\sigma_{x}=0$ and (random) crystal-field coupling $\Delta$, in comparison to the well established picture of the standard Ising ferromagnet. Although there is no doubt that the local order parameter of most interest is $\left\{Q_{x}\right\}$, yet, it can not distinguish between random distributions of $\sigma_{x}= \pm 1$ and regions of $\sigma_{x}=0$. To bring out this distinction, we present in Fig. 5 configurations of the dynamic quadrupole moment $\left\{O_{x}\right\}$ over a full cycle of the external field, where $O=\frac{1}{2 t_{1 / 2}} \oint \rho(t) d t$ and $\rho(t)=1-\frac{1}{N} \sum_{x=1}^{N} \sigma_{x}^{2}$ denotes the order parameter conjugate to the crystal-field coupling $\Delta$. Moreover, in analogy to Figs. 2 and 3, the additional Figs. 6 and 7 present the time evo- 
lution of $\rho(t)$ and the period dependencies of the quadrupole moment of the kinetic random $p=1 / 2$ Blume-Capel model. In all Figs. 5 - 7 simulation parameters are exactly the same to those used in Figs. 2 - 4 above.

Of course, the dynamic quadrupole moment is always 0 for the kinetic spin- $1 / 2$ Ising model, because $\sigma_{x}= \pm 1$ in this case. For the spin-1 Blume-Capel model the density of vacancies is controlled by the crystal-field coupling $\Delta$ and, thus, the value of $O$ changes depending on $\Delta$. When the value of $\Delta$ increases, starting from the Ising limit $(\Delta \rightarrow-\infty)$, the number of vacancies increases as well in the system, so that $O$ tends to increase from its minimum value. For the case of the kinetic random $p=1 / 2$ Blume-Capel model at $\Delta=1$, as depicted in Figs. 4 and 5 , one may conclude that the effect of vacancies is not significant. Moreover, for this particular case of $\Delta=1$ we have performed a quantitative comparison among the pure $(p=0)$ and random $(p=1 / 2)$ model and did not observe any significant differences in the configurations of the local dynamic order parameter $Q$ and quadrupole moment $O$ that are worth to be noted. On the other hand, we expect to see more prominent effects in the small- $p$ and high- $\Delta$ limits that correspond to the ex-first-order transition regime of the equilibrium model's phase diagram [51]. In fact, the set of parameters $p=0.02$ and $\Delta=2$ may be a promising choice and we present in Figs. 8 and 9 three sets of configurations for both the local dynamic order parameter and quadrupole moment, below, around, and above the dynamic phase transition. These snapshots fully corroborate our claim that in this regime the underlying phenomena are indeed controlled by the vacancies, as expected.

To further explore the nature of dynamic phase transitions encountered in the above disordered kinetic model we performed a finite-size scaling analysis based on the observables outlined in Sec. IIC, Previous studies in the field indicated that although finite-size scaling is a tool that has been designed for the study of equilibrium phase transitions, it can be successfully applied as well to far from equilibrium systems [28 32].

As an illustrative example we present in Fig. 10 the finite-size behavior of the dynamic order parameter (main panel) and the emerging susceptibility (inset) - see also Eq. (6) - for the case $\Delta=1$ and for two characteristic system sizes. The dynamic order parameter starts off from a finite value and approaches zero as the half period increases, showing a sharp change for a range of $t_{1 / 2}$ values that correspond to the respective peak in the dynamic susceptibility. These maxima locations of $\chi_{L}^{Q}$, denoted hereafter as $\left(\chi_{L}^{Q}\right)^{*}$, may be used to define suitable pseudocritical half periods $t_{1 / 2}^{*}$. In full analogy we may also denote the 
heat-capacity maxima as $\left(\chi_{L}^{E}\right)^{*}$.

We start the presentation of our finite-size scaling analysis with a characteristic determination of the critical half period $t_{1 / 2}^{\mathrm{c}}$ and the exponent $\nu$ for the system with $\Delta=1$. A similar analysis was performed for the other values of $\Delta$ as well and a summary of our findings is given in Tab. II. The main panel of Fig. 11 illustrates the shift behavior of the peak locations $t_{1 / 2}^{*}$ of the dynamic susceptibility and heat capacity as a function of $1 / L$. The solid lines are a joint fit of the form [63 65]

$$
t_{1 / 2}^{*}=t_{1 / 2}^{\mathrm{c}}+b L^{-1 / \nu}
$$

The obtained values for the critical parameters are $t_{1 / 2}^{\mathrm{c}}=65.96(6)$ and $\nu=1.03(3)$. Clearly, the value of $\nu$ is in very good agreement with the value of $\nu=1$ in the $2 \mathrm{D}$ equilibrium Ising universality class [66].

Additional evidence of universality may be obtained from the fourth-order Binder cumulant $U_{L}^{Q}$ defined in Eq. (8) for the case of the dynamic order parameter. In the inset of Fig. 11 we present our numerical data of $U_{L}^{Q}$ for $\Delta=1$ and a wide range of sizes studied. The vertical dashed line marks the critical half-period value of the system $t_{1 / 2}^{\mathrm{c}}$ as estimated from the analysis of Eq. (9) and the horizontal dashed line the universal value $U^{*}=0.6106924(16)$ of the 2D equilibrium Ising model [67]. Certainly, the crossing point is expected to depend on the lattice size $L$ (as it is also shown in the figure) and the term universal is valid for given lattice shapes, boundary conditions, and isotropic interactions [68, 69]. However, the data shown in the inset of Fig. 11 support, at least qualitatively, another instance of equilibrium Ising universality, since the crossing point is consistent to the value 0.6106924 . We should note here that Hasenbusch et al. presented very strong evidence that the critical Binder cumulant of the equilibrium 2D randomly site-diluted Ising model maintains its pure-system value [70]. In this respect, a dedicated study along the lines of Ref. [70] for an accurate estimation of $U^{*}$ in kinetic random Ising and Blume-Capel models would be welcome.

In this final part we investigate the finite-size scaling behavior of the dynamic susceptibility and heat-capacity maxima. In particular we present in Fig. 12 the size evolution of the dynamic susceptibility peaks in a log-log scale for all three values of $\Delta$ considered. The solid lines are a fit of the form [71]

$$
\left(\chi_{L}^{Q}\right)^{*} \sim L^{\gamma / \nu}
$$


providing estimates for the magnetic exponent ratio $\gamma / \nu$ in excellent agreement with the Ising universality class value of $7 / 4$ - see also Tab. I below. At this stage, it would be ideal to also observe the possible double logarithmic scaling behavior of the heat-capacity maxima $\left(\chi_{L}^{E}\right)^{*}$, as predicted by Ref. [72] for the disordered Ising ferromagnet. Indeed, as it is shown in Fig. 13 the data for $L \geq 64$ are fairly good described by a fit of the form

$$
\left(\chi_{L}^{E}\right)^{*} \sim \ln [\ln (L)]
$$

\section{CONCLUSIONS}

We investigated, using extensive Monte Carlo simulations, the effect of quenched disorder in the crystal-field coupling on the dynamic phase transition of the square-lattice BlumeCapel model under a periodically oscillating magnetic field. At a first qualitative level, the role of vacancies and the crystal-field coupling has been scrutinized by examining the configurations of the dynamic order parameter and quadrupole moment of the system for a wide range of simulation parameters. At a second stage, the application of finite-size scaling techniques allowed us to probe with good accuracy the values of critical exponents describing this dynamic phase transition, all of which were found to be compatible with

those of the equilibrium Ising ferromagnet. An additional study of the scaling behavior of the heat capacity revealed a double logarithmic divergence, as expected for the disordered Ising ferromagnet. To conclude, although universality is a cornerstone in the theory of critical phenomena it stands on a less solid foundation for the case of nonequilibrium systems under the presence of quenched disorder. We hope that our contribution will stimulate further research in this direction.

\section{ACKNOWLEDGMENTS}

The authors would like to thank the two anonymous referees for their instructive comments. The numerical calculations reported in this paper were performed at TÜBİTAK ULAKBIM (Turkish agency), High Performance and Grid Computing Center (TRUBA Resources). We also acknowledge the provision of computing time on the parallel computer 
cluster Zeus of Coventry University.

[1] T. Tomé and M.J. de Oliveira, Phys. Rev. A 41, 4251 (1990).

[2] W.S. Lo and R.A. Pelcovits, Phys. Rev. A 42, 7471 (1990).

[3] M.F. Zimmer, Phys. Rev. E 47, 3950 (1993).

[4] M. Acharyya and B.K. Chakrabarti, Phys. Rev. B 52, 6550 (1995).

[5] B.K. Chakrabarti and M. Acharyya, Rev. Mod. Phys. 71, 847 (1999).

[6] M. Acharyya, Phys. Rev. E 56, 1234 (1997).

[7] M. Acharyya, Phys. Rev. E 69, 027105 (2004).

[8] G.M. Buendía and E. Machado, Phys. Rev. E 58, 1260 (1998).

[9] G.M. Buendía and E. Machado, Phys. Rev. B 61, 14686 (2000).

[10] H. Fujisaka, H. Tutu, and P.A. Rikvold, Phys. Rev. B 63, 036109 (2001).

[11] H. Jang, M.J. Grimson, and C.K. Hall, Phys. Rev. E 68, 046115 (2003).

[12] H. Jang, M.J. Grimson, and C.K. Hall, Phys. Rev. B 67, 094411 (2003).

[13] X. Shi, G. Wei, and L. Li, Phys. Lett. A 372, 5922 (2008).

[14] A. Punya, R. Yimnirun, P. Laoratanakul, and Y. Laosiritaworn, Physica B 405, 3482 (2010).

[15] P. Riego and A. Berger, Phys. Rev. E 91, 062141 (2015).

[16] M. Keskin, O. Canko, and U. Temizer, Phys. Rev. E 72, 036125 (2005).

[17] M. Keskin, O. Canko, and Ü. Temizer, J. Exp. Theor. Phys. 104, 936 (2007).

[18] D.T. Robb, P.A. Rikvold, A. Berger, and M.A. Novotny, Phys. Rev. E 76, 021124 (2007).

[19] B. Deviren and M. Keskin, J. Magn. Magn. Mater. 324, 1051 (2012).

[20] Y. Yüksel, E. Vatansever, and H. Polat, J. Phys.: Condens. Matter 24, 436004 (2012).

[21] Y. Yüksel, E. Vatansever, U. Akinci, and H. Polat, Phys. Rev. E 85, 051123 (2012).

[22] E. Vatansever, Phys. Lett. A 381, 1535 (2017).

[23] Y.-L. He and G.-C. Wang, Phys. Rev. Lett. 70, 2336 (1993).

[24] D.T. Robb, Y.H. Xu, O. Hellwig, J. McCord, A. Berger, M.A. Novotny, and P.A. Rikvold, Phys. Rev. B 78, 134422 (2008).

[25] J.-S. Suen and J.L. Erskine, Phys. Rev. Lett. 78, 3567 (1997).

[26] A. Berger, O. Idigoras, and P. Vavassori, Phys. Rev. Lett. 111, 190602 (2013).

[27] P. Riego, P. Vavassori, and A. Berger, Phys. Rev. Lett. 118, 117202 (2017). 
[28] S.W. Sides, P.A. Rikvold, and M.A. Novotny, Phys. Rev. Lett. 81, 834 (1998).

[29] S.W. Sides, P.A. Rikvold, and M.A. Novotny, Phys. Rev. E 59, 2710 (1999).

[30] G. Korniss, C.J. White, P.A. Rikvold, and M.A. Novotny, Phys. Rev. E 63, 016120 (2000).

[31] G.M. Buendía and P.A. Rikvold, Phys. Rev. E 78, 051108 (2008).

[32] H. Park and M. Pleimling, Phys. Rev. E 87, 032145 (2013).

[33] H. Park and M. Pleimling Phys. Rev. Lett. 109, 175703 (2012).

[34] K. Tauscher and M. Pleimling, Phys. Rev. E 89, 022121 (2014).

[35] G.M. Buendía and P.A. Rikvold, Phys. Rev. B 96, 134306 (2017).

[36] E. Vatansever and N.G. Fytas, Phys. Rev. E 97, 012122 (2018).

[37] E. Vatansever and N.G. Fytas, Phys. Rev. E 97, 062146 (2018).

[38] P.A. Rikvold and M. Kolesik, J. Phys. A: Math. Gen. 35, L117 (2002).

[39] X. Shi and G. Wei, Phys. Scr. 89, 075805 (2014).

[40] M. Acharyya and A. Halder, J. Magn. Magn. Mater. 426, 53 (2017).

[41] G. Grinstein, C. Jayaprakash, and Y. He, Phys. Rev. Lett. 55, 2527 (1985).

[42] G. Gulpinar and E. Vatansever, J. Stat. Phys. 146, 787 (2012).

[43] G. Gulpinar, E. Vatansever, and M. Agartioglu, Physica A 391, 3574 (2012).

[44] U. Akinci, Y. Yüksel, E. Vatansever, and H. Polat, Physica A 391, 5810 (2012).

[45] E. Vatansever, U. Akinci, and H. Polat, J. Magn. Magn. Mater. 344, 89 (2013).

[46] E. Vatansever, U. Akinci, Y. Yüksel, and H. Polat, J. Magn. Magn. Mater. 329, 14 (2013).

[47] E. Vatansever and H. Polat, Phys. Lett. A 379, 1568 (2015).

[48] M. Blume, Phys. Rev. 141, 517 (1966); H.W. Capel, Physica (Utr.) 32, 966 (1966); 33, 295 (1967); 37, 423 (1967).

[49] N.S. Branco and B.M. Boechat, Phys. Rev. B 56, 11673 (1997).

[50] Sumedha and S. Mukherjee, Phys. Rev. E 101, 042125 (2020).

[51] E. Vatansever, Z.D. Vatansever, P.E. Theodorakis, and N.G. Fytas, Phys. Rev. E 102, 062138 (2020).

[52] C. Buzano, A. Maritan, and A. Pelizzola, J. Phys. Condens. Matter 6, 327 (1994).

[53] C.J. Silva, A.A. Caparica, and J.A. Plascak, Phys. Rev. E 73, 036702 (2006).

[54] A. Malakis, A.N. Berker, I.A. Hadjiagapiou, N.G. Fytas, and T. Papakonstantinou, Phys. Rev. E 81, 041113 (2010).

[55] W. Kwak, J. Jeong, J. Lee, and D.-H. Kim, Phys. Rev. E 92, 022134 (2015). 
[56] J. Zierenberg, N.G. Fytas, M. Weigel, W. Janke, and A. Malakis, Eur. Phys. J. Special Topics 226, 789 (2017).

[57] N. Metropolis, A.W. Rosenbluth, M.N. Rosenbluth, A.H. Teller, and E. Teller, J. Chem. Phys. 21, 1087 (1953).

[58] D.P. Landau and K. Binder, A Guide to Monte Carlo Simulations in Statistical Physics (Cambridge University Press, Cambridge, U.K., 2000).

[59] M.E.J. Newman and G.T. Barkema, Monte Carlo Methods in Statistical Physics (Oxford University Press, New York, 1999).

[60] R.J. Glauber, J. Math. Phys. 4 , 294 (1963).

[61] W.H. Press, S.A. Teukolsky, W.T. Vetterling, and B.P. Flannery, Numerical Recipes in C, 2nd ed. (Cambridge University Press, Cambridge, 1992).

[62] K. Binder, Z. Phys. B: Condens. Matter 43, 119 (1981); Phys. Rev. Lett. 47, 693 (1981).

[63] M.E. Fisher, Critical Phenomena, edited by M.S. Green (Academic, London, 1971).

[64] V. Privman, Finite Size Scaling and Numerical Simulation of Statistical Systems (World Scientific, Singapore, 1990).

[65] K. Binder, Computational Methods in Field Theory, edited by C.B. Lang and H. Gausterer (Springer, Berlin, 1992).

[66] A. E. Ferdinand and M. E. Fisher, Phys. Rev. 185, 832 (1969).

[67] J. Salas and A.D. Sokal, J. Stat. Phys. 98, 551 (2000).

[68] W. Selke, J. Stat. Mech.: Theor. Exp. (2007) P04008.

[69] W. Selke and L.N. Shchur, Phys. Rev. E 80, 042104 (2009).

[70] M. Hasenbusch, F. Parisen Toldin, A. Pelissetto, and E. Vicari, Phys. Rev. E 78, 011110 (2008).

[71] A.M. Ferrenberg and D.P. Landau, Phys. Rev. B 44, 5081 (1991).

[72] Vik.S. Dotsenko and Vl.S. Dotsenko, Sov. Phys. JETP Lett. 33, 37 (1981). 


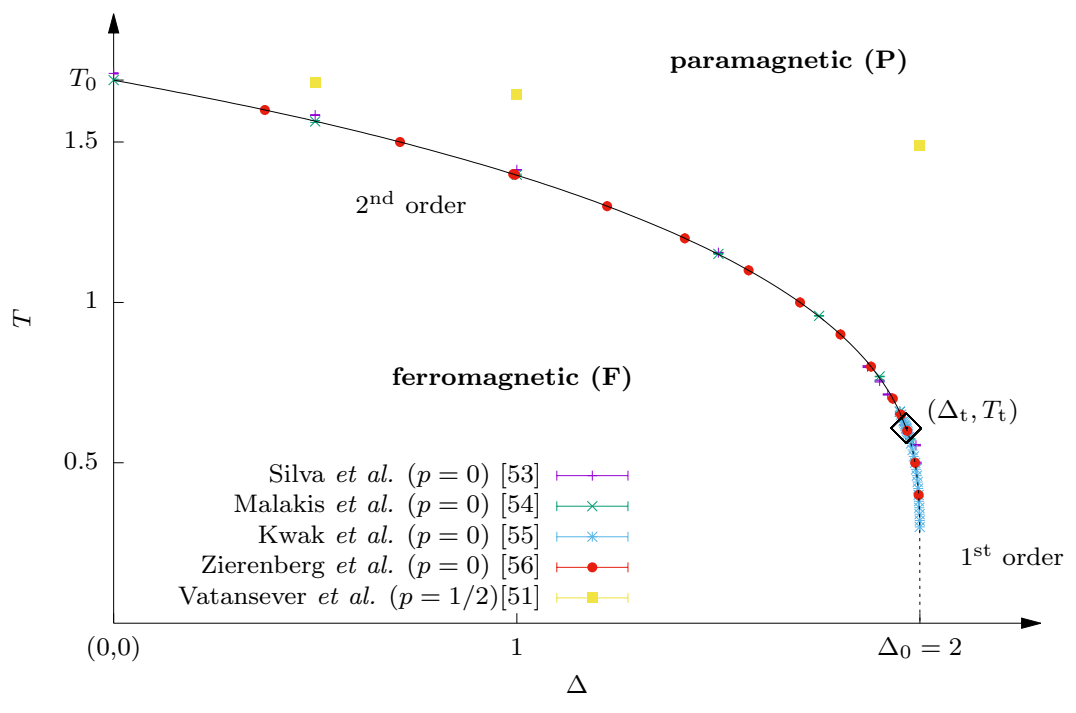

FIG. 1. Phase diagram of the pure $(p=0)$ and random $(p=1 / 2)$ square-lattice Blume-Capel model in the $\Delta-T$ plane showing the ferromagnetic $(\mathbf{F})$ and paramagnetic $(\mathbf{P})$ phases that are separated by a continuous transition at small $\Delta$ (solid line) and a first-order at large $\Delta$ (dotted line). The line segments meet at a tricritical point $\left(\Delta_{\mathrm{t}}, T_{\mathrm{t}}\right)$ marked by a black rhombus. Numerical data shown are selected estimates from previous studies, as indicated also in the panel.

TABLE I. A summary of critical parameters describing the dynamic phase transition of the squarelattice kinetic Blume-Capel model in a quenched random crystal field. Note that the values of $\Delta$ considered in the current work, given the randomness distribution (2) with $p=1 / 2$, correspond to the second-order transition regime of the model's equilibrium phase diagram. One needs very small values of $p$, i.e., $p \leq 0.1$, in order to reach the originally first-order transition regime at high values of $\Delta \approx 2$, see Figs. 9 - 13 in Ref. [51].

\begin{tabular}{|c|c|c|c|}
\hline$\Delta$ & $t_{1 / 2}^{\mathrm{c}}$ & $\nu$ & $\gamma / \nu$ \\
\hline 0.5 & $72.41(9)$ & $1.00(3)$ & $1.75(1)$ \\
\hline 1 & $65.96(6)$ & $1.03(3)$ & $1.76(1)$ \\
\hline 2 & $47.61(7)$ & $1.05(7)$ & $1.75(2)$ \\
\hline
\end{tabular}



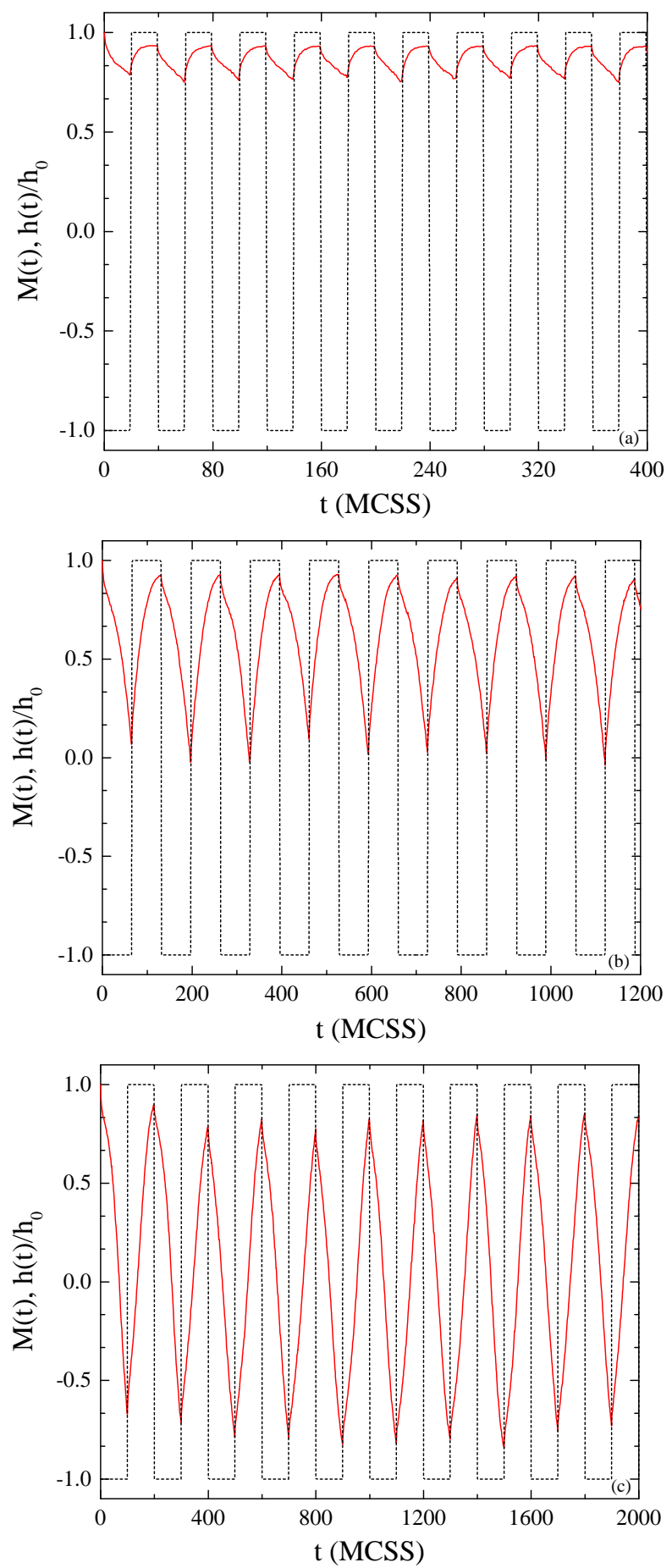

FIG. 2. Time series of the magnetization (red solid curves) of the kinetic random $p=1 / 2$ BlumeCapel model under the presence of a square-wave magnetic field (black dashed lines) for $L=192$ at $\Delta=1$, for three values of the half period of the external field: (a) $t_{1 / 2}=20$ MCSS, corresponding to a dynamically ordered phase, (b) $t_{1 / 2}=66 \mathrm{MCSS}$, close to the dynamic phase transition, and (c) $t_{1 / 2}=100$ MCSS, corresponding to a dynamically disordered phase. Note that for the sake of clarity the ratio $h(t) / h_{0}$ is displayed. 


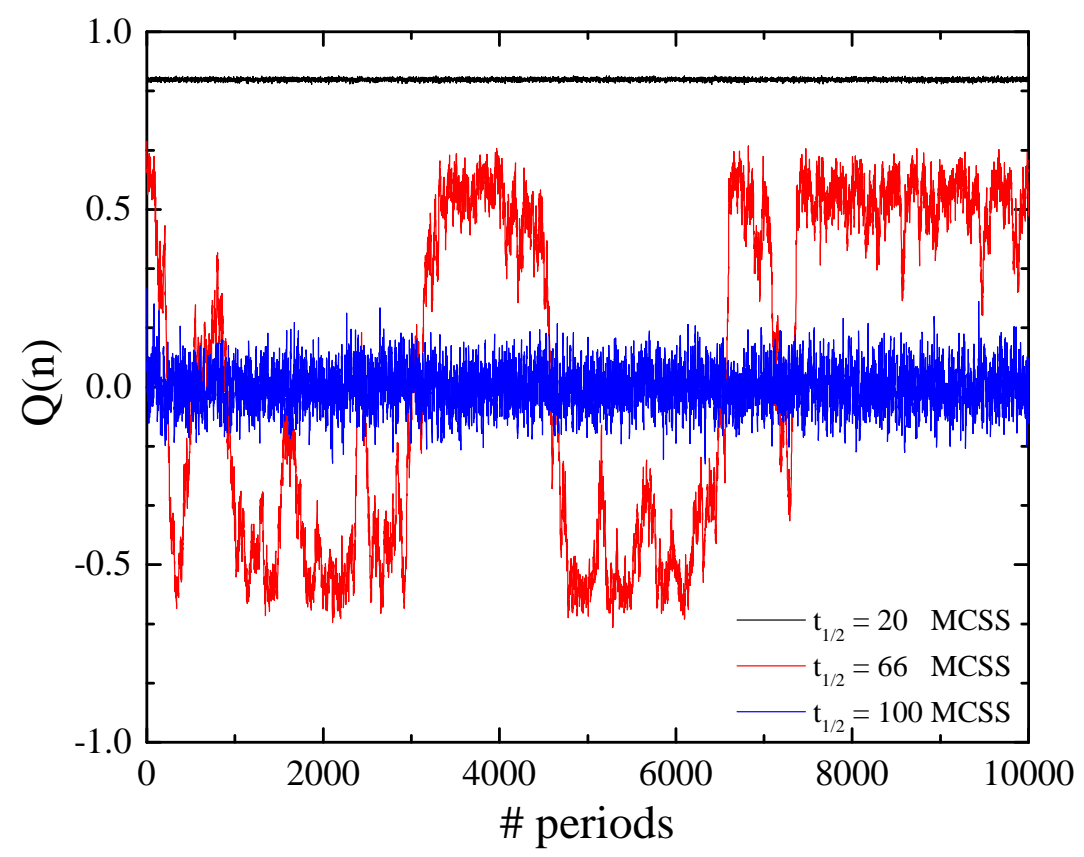

FIG. 3. Period dependencies of the dynamic order parameter of the kinetic random $p=1 / 2$ Blume-Capel model for $L=192$ at $\Delta=1$. Results are shown for the three characteristic cases of the half period of the external field, following Fig. 2 . 

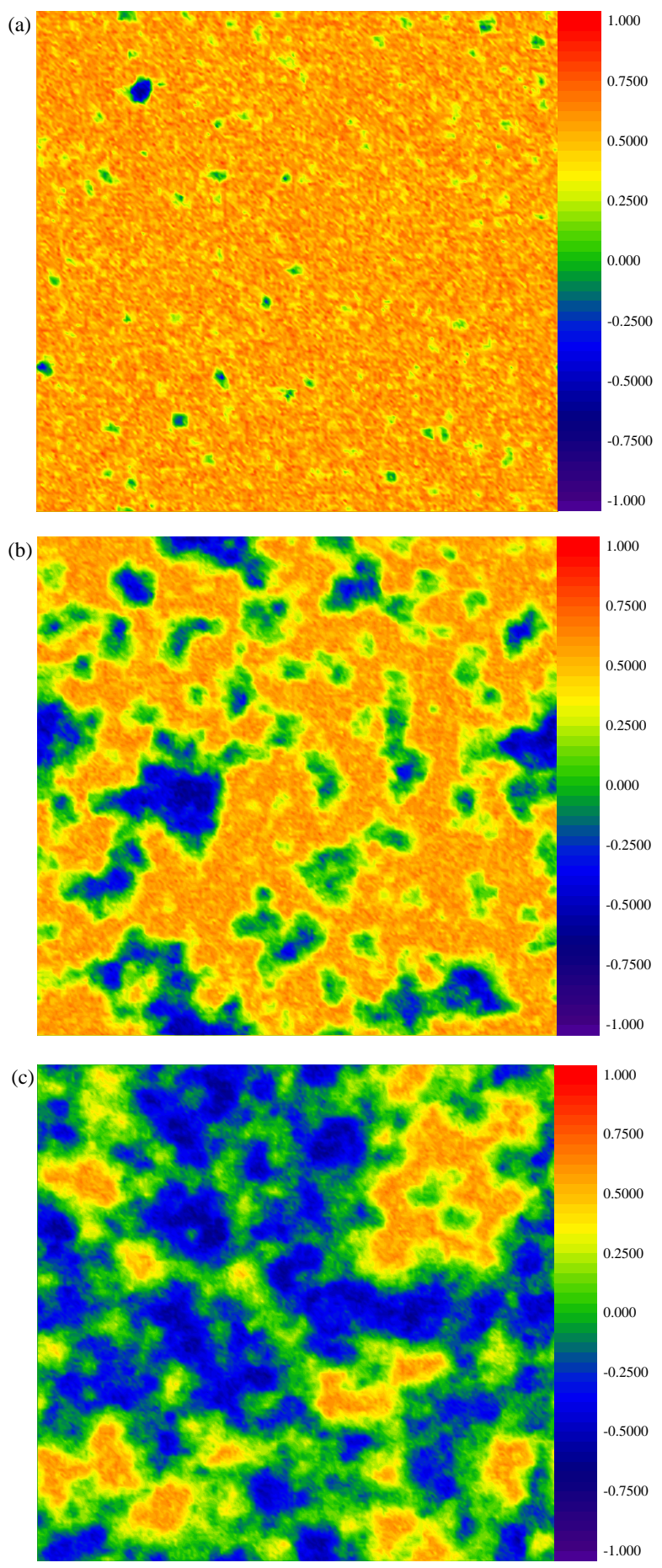

FIG. 4. Configurations of the local dynamic order parameter $\left\{Q_{x}\right\}$ of the kinetic random $p=1 / 2$ Blume-Capel model for $L=192$ at $\Delta=1$. The "snapshots" of $\left\{Q_{x}\right\}$ for each regime are the set of local period-averaged spins during some representative period. Three panels are shown: (a) $t_{1 / 2}=20$ MCSS $<t_{1 / 2}^{\mathrm{c}}-$ dynamically ordered phase, $(\mathrm{b}) t_{1 / 2}=66 \mathrm{MCSS} \approx t_{1 / 2}^{\mathrm{c}}$ - near the dynamic phase transition, and (c) $t_{1 / 2}=100 \mathrm{MCSS}>t_{1 / 2}^{\mathrm{c}}-$ dynamically disordered phase. 

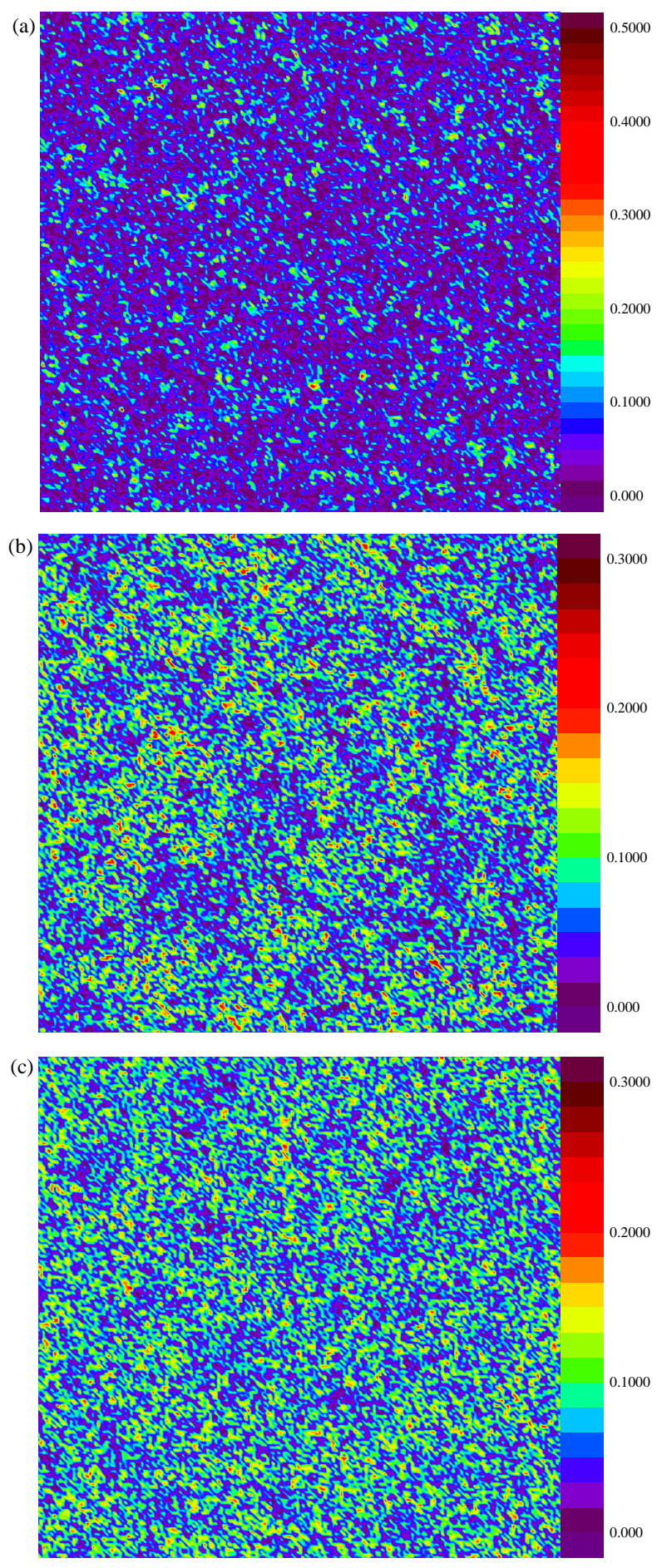

FIG. 5. In full analogy with Fig. 4 we show snapshots of the period-averaged quadrupole moment conjugate to the crystal-field coupling $\Delta$. Simulation parameters are exactly the same as those used in Figs. 4(a)-4(c). 

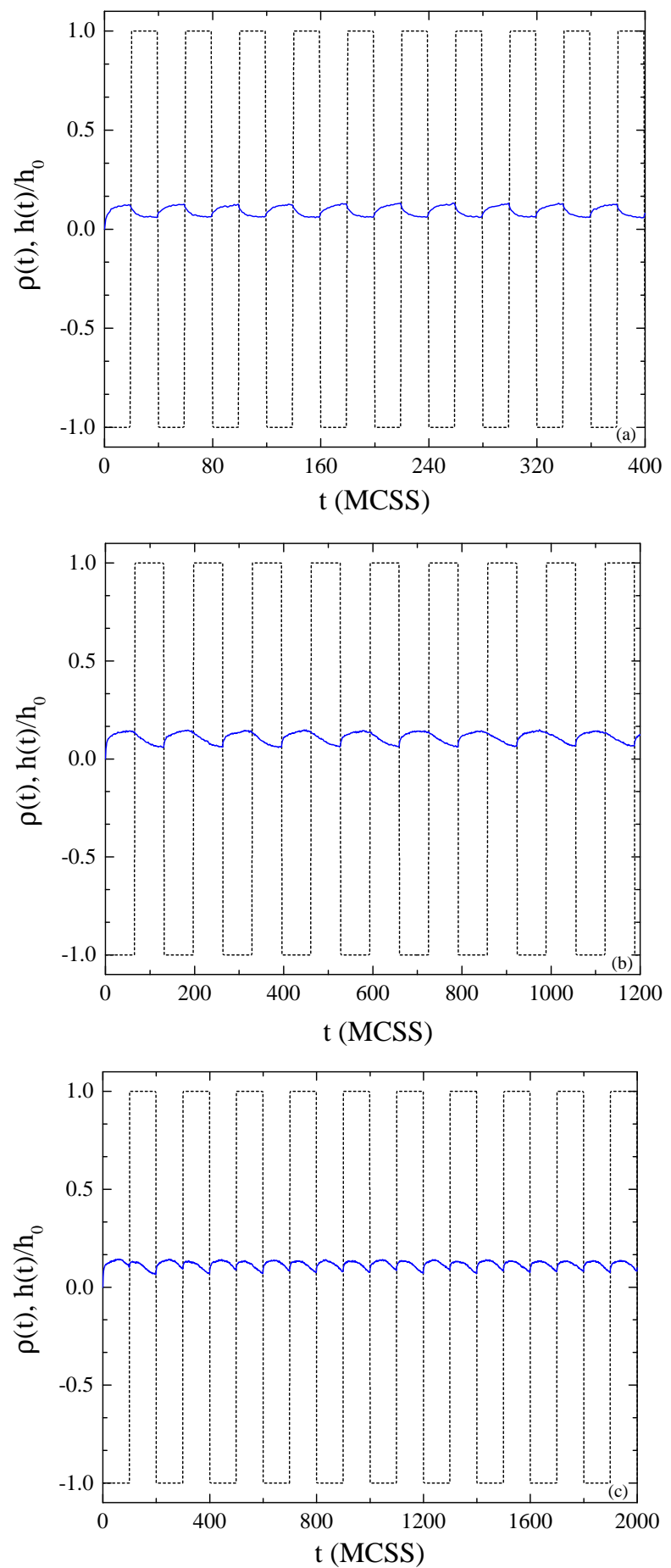

FIG. 6. Time series of the order parameter conjugate to the crystal field $\rho(t)$ (solid blue curves) of the kinetic random $p=1 / 2$ Blume-Capel model under the presence of a square-wave magnetic field (black dashed lines) for $L=192$ at $\Delta=1$, for three values of the half period as in Fig. 2a (a) $t_{1 / 2}=20 \mathrm{MCSS}$, (b) $t_{1 / 2}=66 \mathrm{MCSS}$, and (c) $t_{1 / 2}=100 \mathrm{MCSS}$. Again for the sake of clarity the ratio $h(t) / h_{0}$ is displayed. 


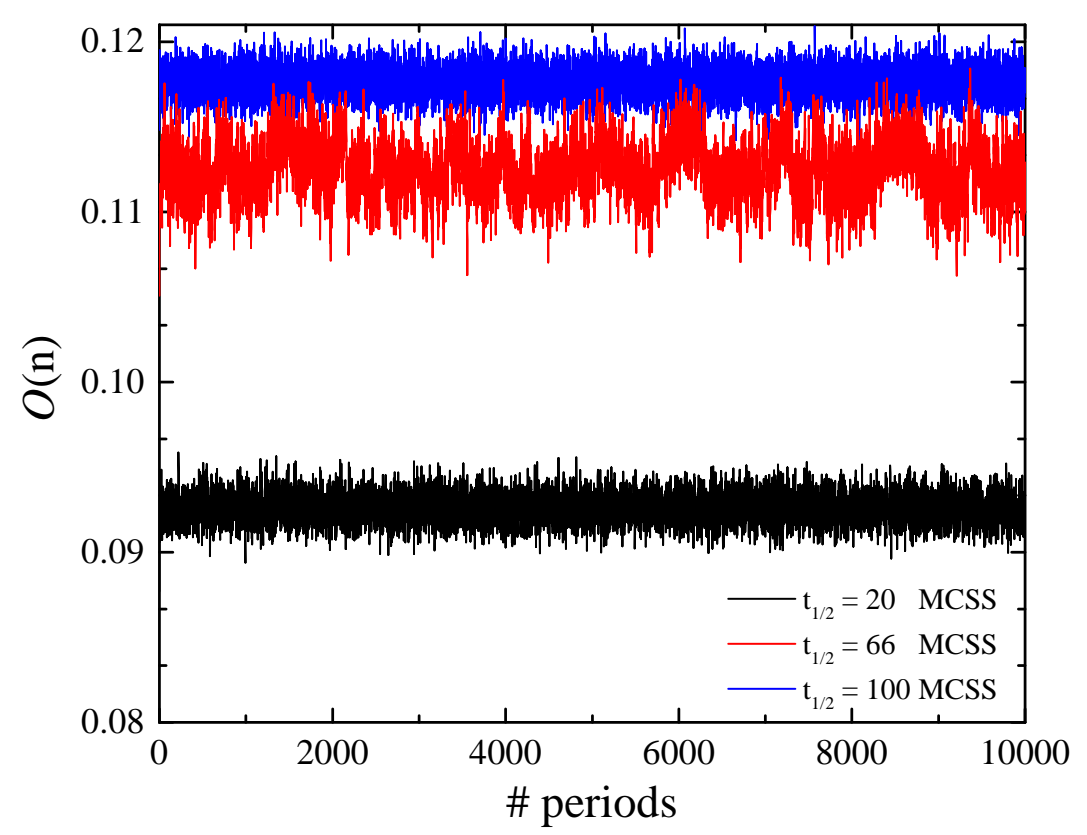

FIG. 7. Period dependencies of the dynamic quadrupole moment $O$ of the kinetic random $p=1 / 2$ Blume-Capel model for $L=192$ at $\Delta=1$. Results are shown for the three characteristic cases of the half period of the external field, following Fig. 6 . 
(a)

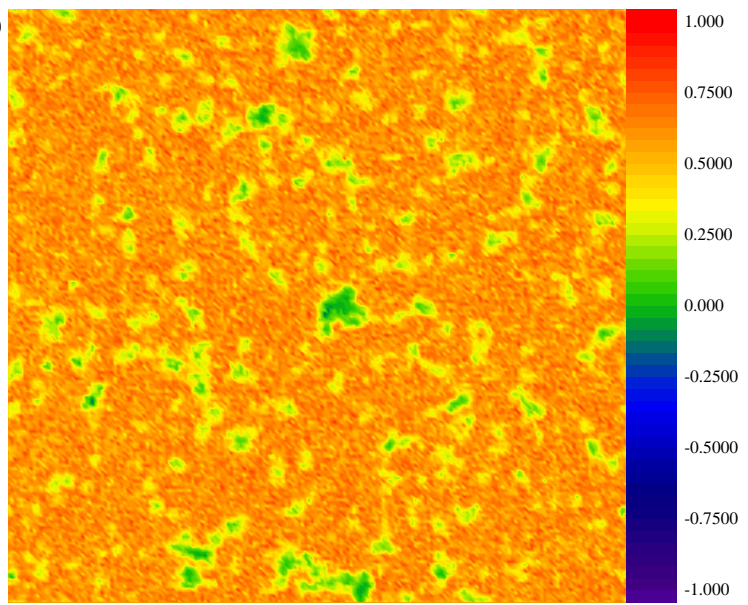

(b)

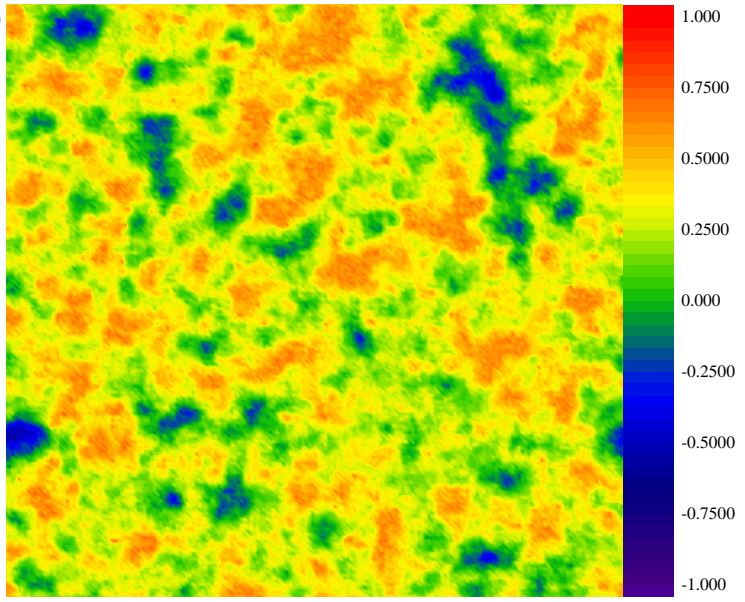

(c)
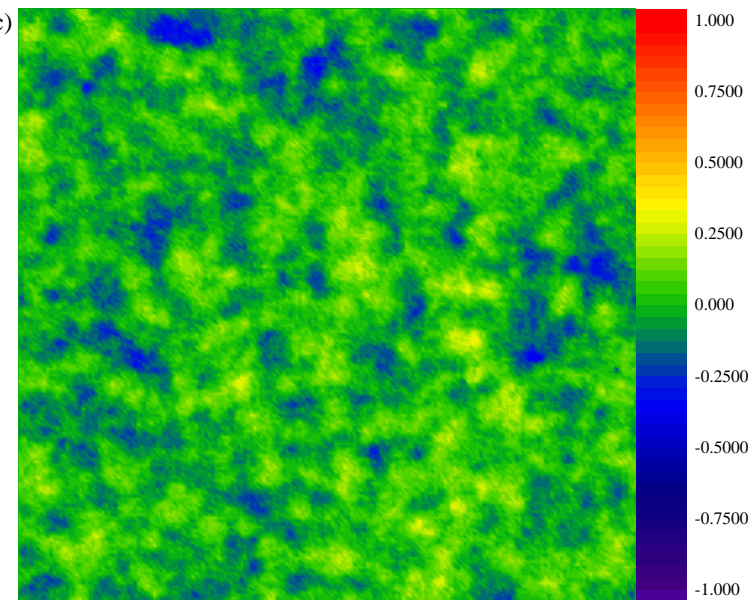

FIG. 8. Configurations of the local dynamic order parameter $\left\{Q_{x}\right\}$ of the kinetic random BlumeCapel model for $L=192, p=0.02$, and $\Delta=2$. Note that for this set of $(p, \Delta)$-parameters we approximated the critical half period of the system to be $t_{1 / 2}^{\mathrm{c}} \approx 53$, from the peak positions of the corresponding dynamic susceptibility and heat-capacity curves. Three panels are shown: (a) $t_{1 / 2}=20$ MCSS $<t_{1 / 2}^{\mathrm{c}}-$ dynamically ordered phase, (b) $t_{1 / 2}=53 \mathrm{MCSS} \approx t_{1 / 2}^{\mathrm{c}}-$ near the dynamic phase transition, and (c) $t_{1 / 2}=100 \mathrm{MCSS}>t_{1 / 2}^{\mathrm{c}}-$ dynamically disordered phase. 


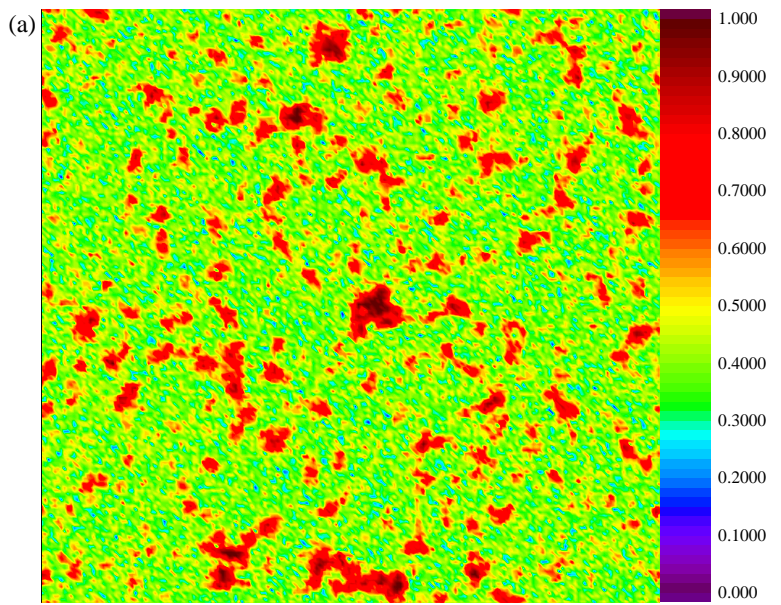

(b)

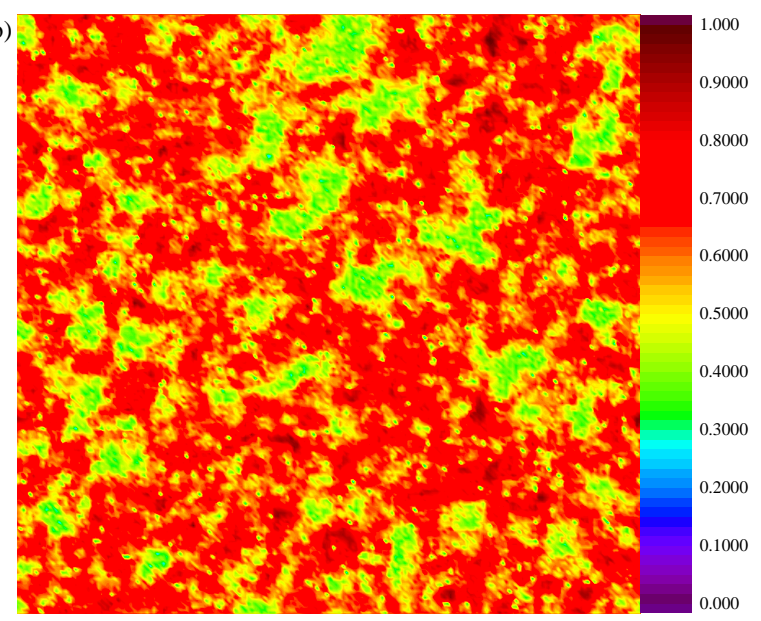

(c)

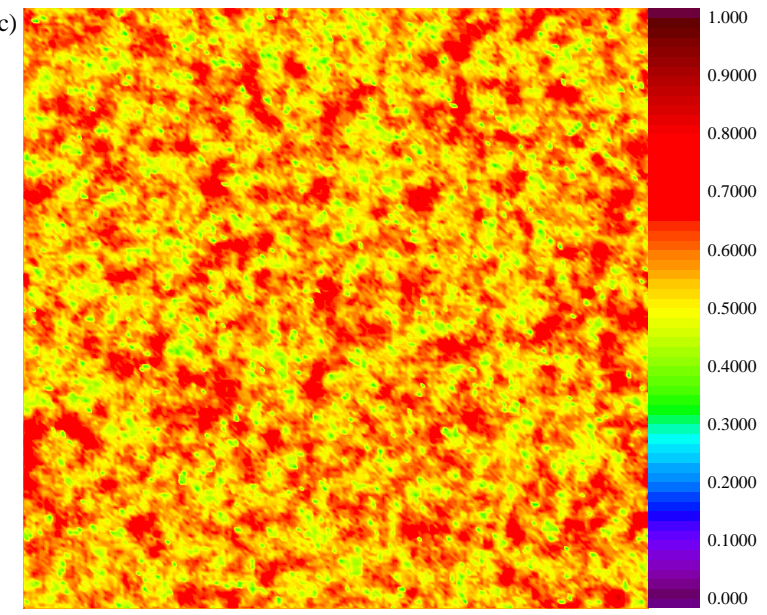

FIG. 9. In full analogy with Fig. 8 we show snapshots of the period-averaged quadrupole moment conjugate to the crystal-field coupling $\Delta$. Simulation parameters are exactly the same as those used in Figs. $8(a)-8(c)$. 


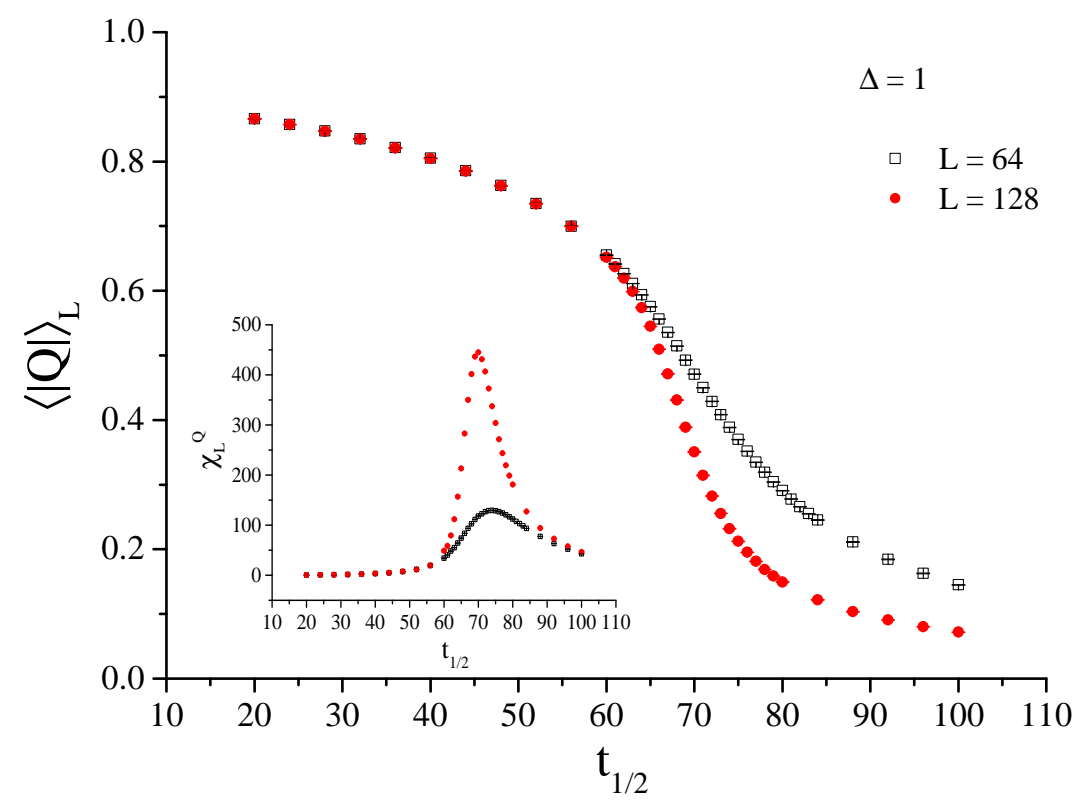

FIG. 10. Typical dynamic order parameter $\langle|Q|\rangle_{L}$ (main panel) and susceptibility $\chi_{L}^{Q}$ (inset) curves of the kinetic random $p=1 / 2$ Blume-Capel model at $\Delta=1$ and for two systems with linear sizes $L=64$ (open black squares) and $L=128$ (filled red circles).

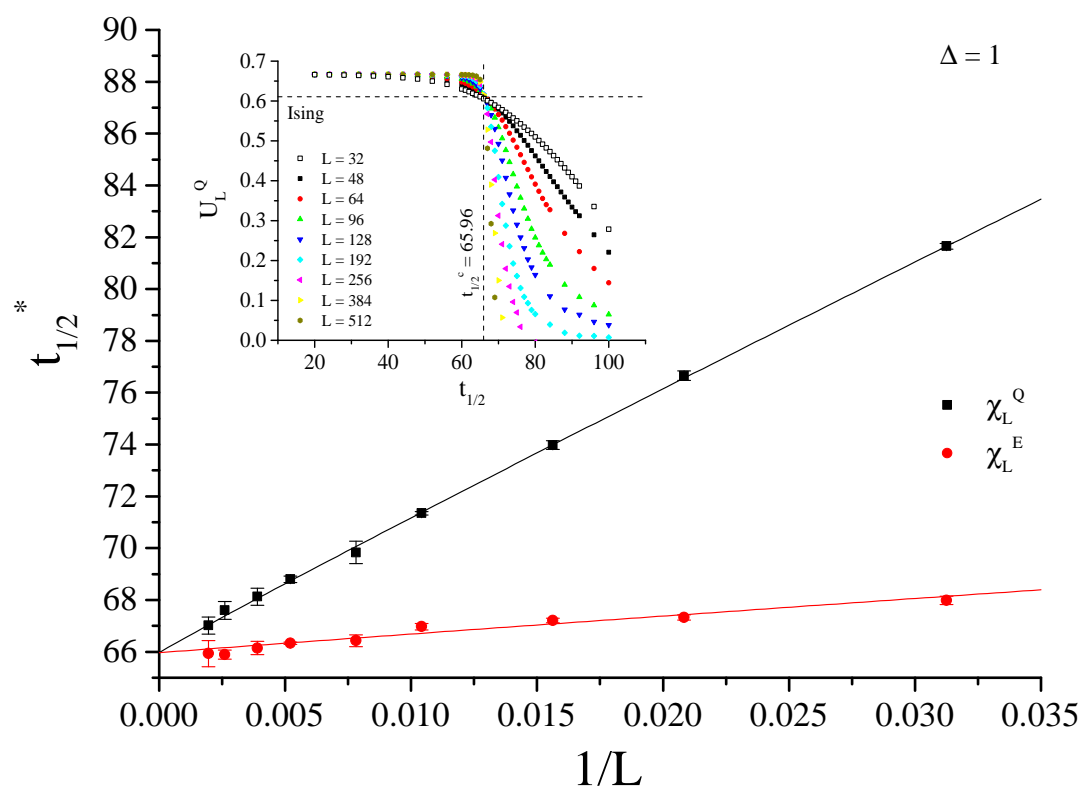

FIG. 11. Shift behavior of the two pseudocritical half periods $t_{1 / 2}^{*}$ corresponding to the maxima of the dynamic susceptibility (filled black squares) and heat capacity (filled red circles) of the kinetic random $p=1 / 2$ Blume-Capel model at $\Delta=1$. The inset illustrates the half-period dependency of the corresponding fourth-order Binder cumulant $U_{L}^{Q}$. 


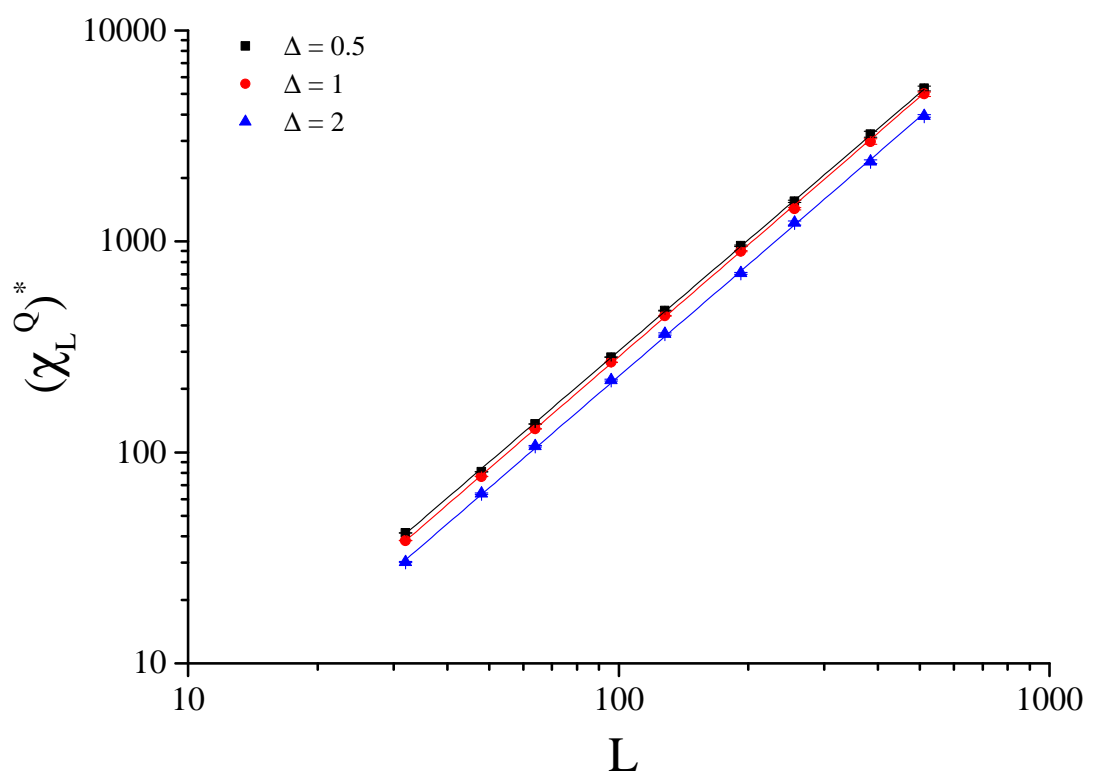

FIG. 12. Finite-size scaling behavior of the dynamic susceptibility maxima $\left(\chi_{L}^{Q}\right)^{*}$ of the kinetic random $p=1 / 2$ Blume-Capel model. Results for three values of $\Delta$ are shown in a log-log scale.

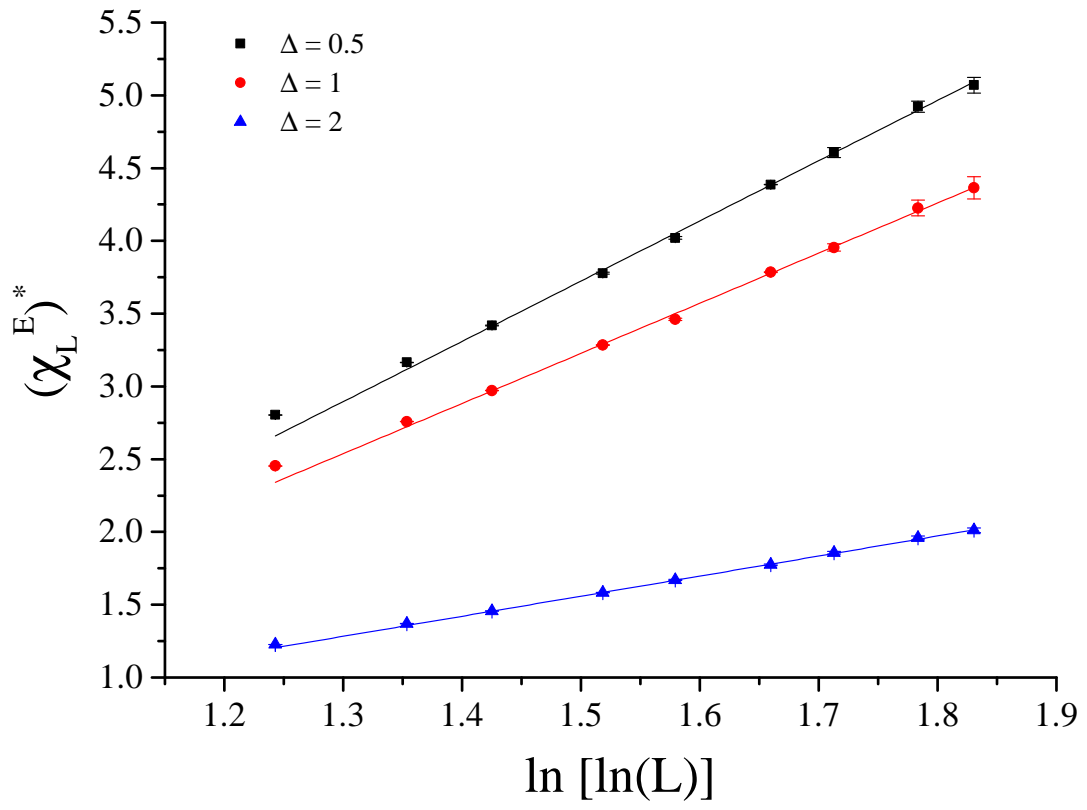

FIG. 13. Double logarithmic scaling behavior of the heat-capacity maxima $\left(\chi_{L}^{E}\right)^{*}$ of the kinetic random $p=1 / 2$ Blume-Capel model for three values of $\Delta$, as indicated in the panel. 